\title{
Evaluation of Thyroid Function Status in Sonographically Suggested Fatty Liver Subjects
}

Farhana Rahman, Sanowar Hossain, Shankar Kumar Biswas, Fatema Sultana Haque, Rubina Begum, Mohana Hossain and Sadia Hossain

Institute of Nuclear Medicine \& Allied Sciences, Dhaka Medical College Hospital Campus, Dhaka, Bangladesh

Correspondence Address : Dr. Farhana Rahman, Senior Medical Officer, Institute of Nuclear Medicine \& Allied Sciences, Dhaka Medical College Hospital Campus, Dhaka, E mail: dr.farhananmc@ gmail.com

\begin{abstract}
Objectives: The liver metabolizes thyroid hormones and thereby influences the regulation of their systematic endocrine effects. On the other hand, thyroid hormones play an important role in hepatic lipid homeostasis. Several studies addressed the association between thyroid function tests and non alcoholic fatty liver disease. The results of those studies indicate that hypothyroidism might be related to non alcoholic fatty liver disease. The recognized link between hypothyroidism and elements of metabolic syndrome may explain this relation. The present study was performed to evaluate the thyroid function status with the severity of sonographically suggested fatty liver subjects.
\end{abstract}

Materials and Methods: This cross sectional study was carried out at the Institute of Nuclear Medicine and Allied Sciences (INMAS) of Dhaka Medical College Hospital Campus, Dhaka during the period of January 2014 to December 2014. A total number of 155 consecutive patients having sonographically detected fatty liver from the above mentioned hospitals were included in this study and they were divided into three grades depending on the sonographic criteria. Data were analyzed between these three grades in respect of age, sex, BMI, thyroid hormone levels (FT3, FT4 and TSH), fasting lipid profile and serum liver enzyme levels. Results: $82(52.9 \%)$ patients had grade 1, $50(32.3 \%)$ had grade 2 and $23(14.8 \%)$ had grade 3 fatty liver. The mean age was found 39 \pm 10.9 years in Grade 1, 39.5 \pm 10.4 years in Grade 2 and 41.4 \pm 7.1 years in Grade 3. The difference was not statistically significant (p>0.05) among three groups. Male to female ratio was almost $2: 3$ in the whole study subjects. The mean BMI was significantly higher in grade $3(p<0.05)$ followed by grade 2 and grade 1. $128(82.6 \%)$ patients were euthyroid, $23(14.8 \%)$ were subclinical hypothyroid and $4 \mathbf{( 2 . 6 \% )}$ were hypothyroid. In Anova test, the mean FT3 and FT4 were significantly declined $(p<0.05)$ with increased grade but the mean TSH level was significantly increased $(p=0.001)$ with increased grade of fatty liver. The Spearman's rank correlation test shows that no significant but a negative correlation $(r=-0.101 ; p=0.175)$ was found between FT3 and fatty liver of the study patients, but a significant negative correlation $(r=-0.277 ; p=0.001)$ was observed between FT4 and fatty liver of the study patients. On the other hand a significant positive correlation $(r=0.325 ; p=0.001)$ was observed between TSH and fatty liver of the study patients. Conclusion: In this study it has been found that thyroid hormone levels are significantly decreased with increasing grades of fatty liver detected by ultrasound. The underlying pathophysiology for this association is still not clear, however several mechanisms have been proposed. So the findings of this study will inspire other researchers to evaluate the thyroid function status with the severity of sonographically suggested fatty liver in larger scale.

Key Words: Fatty liver disease, Thyroid hormone, Metabolic syndrome, Ultrasonogram.

\section{INTRODUCTION}

Fatty liver or hepatic steatosis is a pathological condition characterized by abnormal excessive/ extensive accumulation of lipids mainly triglyceride in liver. When accumulation of lipid becomes chronic, it induces pressure effect on the architecture of liver; produces inflammation, fibrosis and cirrhosis of liver, leading to a chronic condition known as non-alcoholic steatohepatitis (NASH). Simple steatosis (fatty liver), which rarely has any sequelae, is also a part of the condition termed as non-alcoholic fatty liver disease (NAFLD) (1). To diagnose and manage NAFLD World Gastroenterology Organization (WGO), an association of expert hepatologists across the world, are providing guideline in the form of World Gastroenterology Organization Global Guidelines-Nonalcoholic Fatty Liver Disease and Nonalcoholic Steatohepatitis.

NAFLD is now considered as a hepatic feature of metabolic syndrome. It is a rapidly growing disease in both developed and developing countries and is 
probably the most common cause of abnormal liver function tests worldwide. It is estimated to affect nearly $30 \%$ of general population in western countries

(2). Over the past couple of decades, it has become increasingly clear that NAFLD are now the number 1 cause of liver disease in the western countries. The prevalence of NAFLD has doubled during last 20 years, whereas the prevalence of other chronic liver diseases has remained stable or decreased (3).

The prevalence in Asian countries seems to be lower but is increasing secondary to an increase in burden of diabetes mellitus (DM), metabolic syndrome (MetS) and changing in lifestyle.

Although obesity is the commonest and primary metabolic cause, non alcoholic fatty liver disease may arise secondary to several other endocrine disorders, including thyroid dysfunction, growth hormone deficiency, adrenal insufficiency and polycystic ovary syndrome. The prevalence of hypothyroidism in patients with nonalcoholic fatty liver disease is twice that in patients with other chronic liver disease (15\% v $7.2 \%)$ (4).

Several studies have investigated the association between thyroid hormone status and fatty liver disease. The results of those studies indicate that hypothyroidism might be related to hepatic steatosis because there are some common clinical and biochemical features of central obesity, insulin resistance, hypertension and hypertriglyceridemia observed in both NAFLD and hypothyroidism. But few studies revealed no significant association. The results of those previous studies on the role of fatty liver disease in thyroid abnormalities are inconsistent and there is no conclusive evidence about this.

In a developing country like Bangladesh, fatty liver disease is rapidly increasing and thyroid disorders are also common. There is no evidence based information available regarding the relation of thyroid hormone status with the severity of fatty liver disease in Bangladesh. So this study was designed to evaluate the thyroid function status in sonographically suggested fatty liver and to establish the necessity for investigation of thyroid function test in fatty liver subjects.

\section{MATERIALS AND METHODS}

This cross sectional study was carried out at the Institute of Nuclear Medicine and Allied Sciences (INMAS) of Dhaka Medical College Hospital Campus, Dhaka during the period of January 2014 to December 2014. Total 155 subjects were included by non-random purposive sampling. Sonographically detected fatty liver subjects aged 18 years or more were included. But patients with known thyroid disease, current or past history of thyroid hormone or antithyroid drug intake, hepatitis including liver cirrhosis, history of alcohol intake, any hepatotoxic drug intake, associated with any chronic debilitating illness and pregnant women were excluded from the study. All relevant data collected during the investigations were recorded on a pre-designed data collection sheet for each patient.

Abdominal ultrasound scanning was performed in all participants by a skilled sonologist, who was blind to all clinical and laboratory data of patients, using Accuvix V20 scanner, Samsung Medison (South Korea) with a $3.5-\mathrm{MHz}$ curvilinear transducer. US examinations were performed after 6 hours fasting. Each subject was examined in the supine and left lateral positions during quiet inspiration. The presence of grading of fatty infiltration of the liver were recorded: Grade 1: Slight, diffuse increase in fine echoes in liver parenchyma with normal visualization of diaphragm and intrahepatic vessel borders, Grade 2: Moderate, diffuse increase in fine echoes with slightly impaired visualization of intrahepatic vessels and diaphragm, Grade 3: Marked, diffuse increase in fine echoes with poor or non visualization of the intrahepatic vessel borders, diaphragm and posterior right lobe of liver.

Concentration of free triiodothyronine (FT3) and free thyroxin (FT4) were analyzed by radioimmunoassay 
and thyroid stimulating hormone (TSH) was analyzed by immunoradiometric assay. Normal reference laboratory values for FT3: 2.80-9.50 p mol/L, FT4: 9.50-25.50 p mol/L and TSH: 0.3-5.0 m IU/L.

Statistical analysis was performed using SPSS package 16.0 (SPSS Inc, Chicago, Illinois, USA). Categorical data was expressed in percentage or number. Parametric data was expressed in mean \pm SD. Parametric data was evaluated by ANOVA test. Categorical data was evaluated by Chi square test. Significance was defined by $\mathrm{p}$ value $\leq 0.05$. Spearman's rank correlation analysis was used to assess the correlation between variables.

Ethical consideration: This study was approved by the Medical Research Ethics Committee (MREC) of the National Institute of Nuclear Medicine and Allied Sciences, BSMMU campus, Shahbag, Dhaka.

\section{RESULTS}

Out of 155 fatty liver subjects $82(52.9 \%)$ were in grade $1,50(32.3 \%)$ were grade 2 and 23 (14.8\%) were grade 3 (Table 1). Male to female ratio was almost 2:3 in the whole study subjects (Table 2). The mean age was found $39 \pm 10.9$ years in Grade 1, $39.5 \pm 10.4$ years in Grade 2 and $41.4 \pm 7.1$ years in Grade 3 (Table 3). The mean BMI was found $26.9 \pm 3.6 \mathrm{~kg} / \mathrm{m} 2$ in Grade $1,29.2 \pm 4.5 \mathrm{~kg} / \mathrm{m} 2$ in Grade 2 and $30.0 \pm 4.1 \mathrm{~kg} / \mathrm{m} 2$ in Grade 3 (Table 4). Table 5 shows that mean FT3 and FT4 were significantly decreased with increased grades of fatty liver and mean TSH was increased with increased grade of fatty liver. Table 6 shows 6 patients $(7.3 \%)$ had subclinical hypothyroid in Grade 1, 13 (26.0\%) in Grade 2 and 4 (17.4\%) in Grade 3. 4 hypothyroid subjects were (17.4\%) in Grade 3.

Table 1: Distribution of the study population according to grades of fatty liver $(n=155)$

\begin{tabular}{lcc}
\hline Grade of fatty liver & Number of patients & Percentage \\
\hline Grade-1 (mild) & 82 & 52.9 \\
Grade-2 (moderate) & 50 & 32.3 \\
Grade-3 (marked) & 23 & 14.8 \\
\hline
\end{tabular}

Table 2: Distribution of the study population according to sex in different grades of fatty liver $(n=155)$

\begin{tabular}{lccccccc}
\hline \multirow{3}{*}{ Sex } & \multicolumn{5}{c}{ Fatty liver } & \\
\cline { 2 - 6 } & $\begin{array}{c}\text { Grade 1 } \\
(\mathbf{n = 8 2})\end{array}$ & \multicolumn{2}{c}{$\begin{array}{c}\text { Grade } \mathbf{2} \\
(\mathbf{n = 5 0})\end{array}$} & \multicolumn{2}{c}{$\begin{array}{c}\text { Grade 3 } \\
(\mathbf{n = 2 3 )}\end{array}$} & P value \\
\cline { 2 - 6 } & $\mathbf{n}$ & $\mathbf{\%}$ & $\mathbf{n}$ & $\mathbf{\%}$ & $\mathbf{n}$ & $\mathbf{\%}$ & \\
\hline Male & 36 & 43.9 & 17 & 34.0 & 10 & 43.5 & \multirow{2}{*}{$0.508^{\mathrm{ns}}$} \\
Female & 46 & 56.1 & 33 & 66.0 & 13 & 56.5 & \\
\hline
\end{tabular}

Table 3: Distribution of the study population by age in different grades of fatty liver $(n=155)$

\begin{tabular}{lccccccc}
\hline & \multicolumn{5}{c}{ Fatty liver } & \\
\cline { 2 - 7 } Age (in year) & $\begin{array}{c}\text { Grade 1 } \\
(\mathbf{n = 8 2})\end{array}$ & \multicolumn{2}{c}{$\begin{array}{c}\text { Grade 2 } \\
(\mathbf{n = 5 0 )}\end{array}$} & \multicolumn{2}{c}{$\begin{array}{c}\text { Grade 3 } \\
(\mathbf{n = 2 3 )}\end{array}$} & P value \\
& $\mathbf{n}$ & $\mathbf{\%}$ & $\mathbf{n}$ & $\mathbf{\%}$ & $\mathbf{n}$ & $\mathbf{\%}$ & \\
\hline $18-27$ & 7 & 8.5 & 5 & 10.0 & 0 & 0.0 & \\
$28-37$ & 35 & 42.7 & 19 & 38.0 & 4 & 17.4 & \\
$38-47$ & 21 & 25.6 & 14 & 28.0 & 15 & 65.2 & \\
$48-57$ & 14 & 17.1 & 9 & 18.0 & 4 & 17.4 & \\
$58-67$ & 4 & 4.9 & 3 & 6.0 & 0 & 0.0 & \\
$68-77$ & 1 & 1.2 & 0 & 0.0 & 0 & 0.0 & \\
\hline Mean \pm SD & $39.0 \pm 10.9$ & $39.5 \pm 10.4$ & $41.4 \pm 7.1$ & \multirow{2}{*}{$0.613^{\text {ns }}$} \\
Range (min,max) & 18,77 & & 18,65 & 28,55 & \\
\hline
\end{tabular}

Table 4: Distribution of the study patients according to BMI in different grades of fatty liver $(n=155)$

\begin{tabular}{|c|c|c|c|c|c|c|c|}
\hline & \multicolumn{2}{|c|}{$\begin{array}{c}\text { Grade } 1 \\
(\mathrm{n}=82)\end{array}$} & \multicolumn{2}{|c|}{$\begin{array}{c}\text { Grade } 2 \\
(n=50)\end{array}$} & \multicolumn{2}{|c|}{$\begin{array}{c}\text { Grade } 3 \\
(\mathrm{n}=23)\end{array}$} & \\
\hline & $\mathrm{n}$ & $\%$ & $\mathrm{n}$ & $\%$ & n & $\%$ & \\
\hline$<18.5$ (Low) & 1 & 1.2 & 0 & 0.0 & 0 & 0.0 & \\
\hline $18.5-24.9$ (Normal) & 21 & 25.6 & 8 & 16.0 & 2 & 8.7 & \\
\hline$\geq 25$ (0ver weight) & 60 & 73,2 & 42 & 84.0 & 21 & 913 & \\
\hline Mcan \pm SD & \multicolumn{2}{|l|}{26.} & \multicolumn{2}{|c|}{$29.2 \pm 4.5$} & \multicolumn{2}{|c|}{$30.0 \pm 4.1$} & \multirow{2}{*}{$0.001^{\mathrm{s}}$} \\
\hline Range (min,max) & \multicolumn{2}{|c|}{15.637 .9} & \multicolumn{2}{|c|}{19,45} & \multicolumn{2}{|c|}{$21.2,39$} & \\
\hline
\end{tabular}

Table 5: Distribution of the study population in respect to thyroid hormone levels with grades of fatty liver $(n=155)$

\begin{tabular}{|c|c|c|c|c|}
\hline \multirow{2}{*}{$\begin{array}{l}\text { Thyroid hormone } \\
\text { levels }\end{array}$} & $\begin{array}{c}\text { Grade } 1 \\
(n=82)\end{array}$ & $\begin{array}{c}\text { Grade } 2 \\
(n=50)\end{array}$ & $\begin{array}{c}\text { Grade } 3 \\
(n=23)\end{array}$ & \multirow[t]{2}{*}{$P$ value } \\
\hline & $\operatorname{Mean} \pm$ SD & $\operatorname{Mean} \pm$ SD & Mean \pm SD & \\
\hline FT3 (pmol/L) & $4.93 \pm 1.53$ & $4.92 \pm 1.73$ & $4.27 \pm 1.86$ & \multirow{2}{*}{$0.001^{\mathrm{s}}$} \\
\hline Range (min,max) & $2.8,8.81$ & $2.9,8.9$ & $1.1,8.9$ & \\
\hline FT4 (pmol/L) & $19.51 \pm 3.1$ & $18.73 \pm 3.76$ & $16.1 \pm 5.68$ & \multirow{2}{*}{$0.001^{\mathrm{s}}$} \\
\hline Range (min, $\max )$ & $10.95,24.87$ & $10.39,25.0$ & $3.52,24.22$ & \\
\hline$\overline{\text { TSH (mIU/L) }}$ & $3.21 \pm 1.92$ & $4.68 \pm 3.41$ & $13.74 \pm 23.46$ & \multirow{2}{*}{$0.001^{\mathrm{s}}$} \\
\hline Range (min,max) & $0.58,14.5$ & $0.4,15.79$ & $0.51,84.05$ & \\
\hline
\end{tabular}

Table 6: Distribution of the study population according to thyroid hormone status with grades of fatty liver $(n=155)$

\begin{tabular}{|c|c|c|c|c|c|c|c|}
\hline \multirow{3}{*}{ Thyroid hormone status } & \multicolumn{6}{|c|}{ Fatty liver } & \multirow{3}{*}{$P$ value } \\
\hline & \multicolumn{2}{|c|}{$\begin{array}{c}\text { Grade } 1 \\
(n=82)\end{array}$} & \multicolumn{2}{|c|}{$\begin{array}{c}\text { Grade } 2 \\
(n=50)\end{array}$} & \multicolumn{2}{|c|}{$\begin{array}{c}\text { Grade } 3 \\
(n=23)\end{array}$} & \\
\hline & $\mathrm{n}$ & $\%$ & $\mathbf{n}$ & $\%$ & $\mathrm{n}$ & $\%$ & \\
\hline Subclinical hypothyroid & 6 & 7.3 & 13 & 26.0 & 4 & 17.4 & \\
\hline Hypothyroid & 0 & 0.0 & 0 & 0.0 & 4 & 17.4 & $0.001^{\mathrm{s}}$ \\
\hline Euthyroid & 76 & 92.7 & 37 & 74.0 & 15 & 65.2 & \\
\hline
\end{tabular}


Figure 1 shows no significant negative correlation between FT3 and grades of fatty liver. Correlation between FT4 and grades of fatty liver is showing significant negative correlation between FT4 and grades of fatty liver (Figure 2). Significant positive correlation was found between TSH and grades of fatty liver (Figure 3).

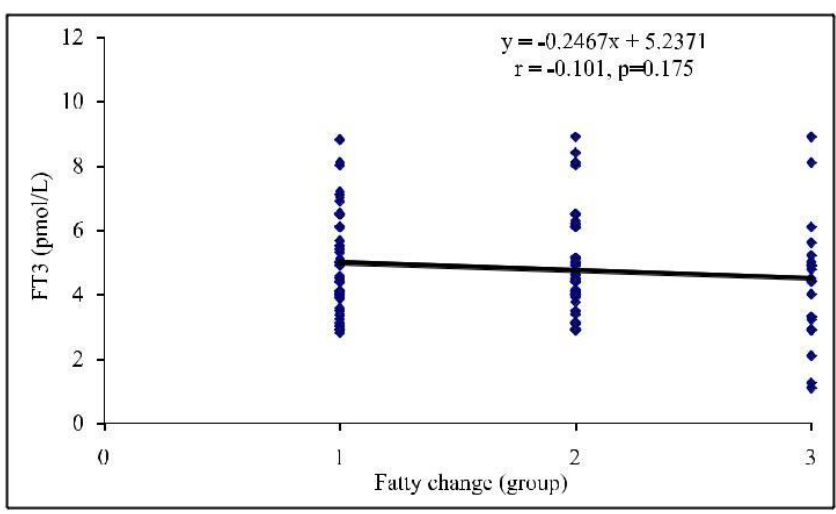

Fig 1: Scatter diagram showing correlation $(r=-0.101 ; p=0.175)$ between FT3 and fatty liver of the study population.

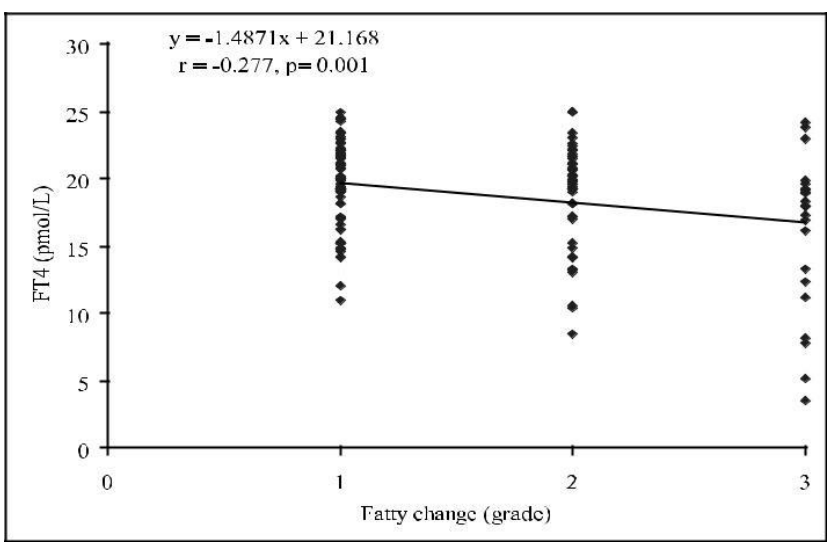

Fig 2: Scatter diagram showing correlation $(r=-0.277 ; p=0.001)$

between FT4 and fatty liver of the study population.

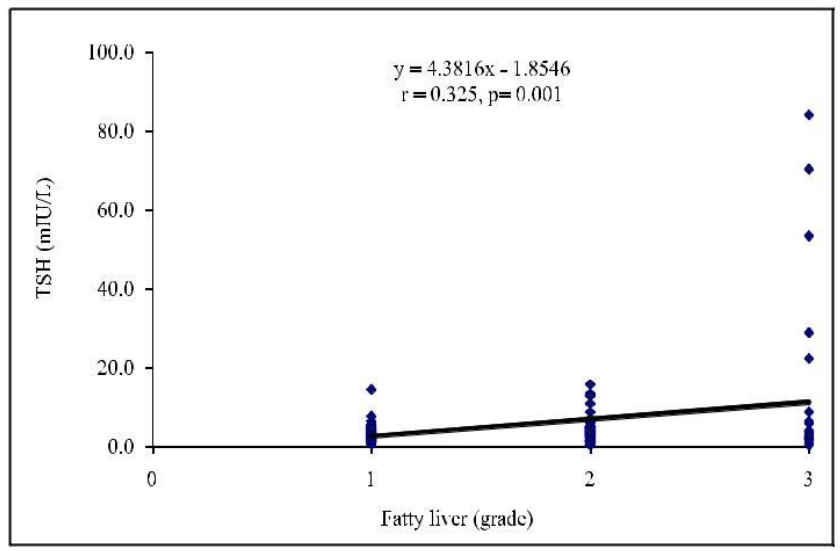

Fig 3: Scatter diagram showing correlation $(r=0.325 ; p=0.001)$ between TSH and fatty liver of the study population.

\section{DISCUSSION}

In this present study it was observed that $82(52.9 \%)$ patients had grade 1 (mild), followed by $32.3 \%$ had grade 2 (moderate) and $14.8 \%$ had grade 3 (marked) fatty liver (Table-1), is in similar with studies among other ethnic groups in Malaysia which reported a lower percent of moderate NAFLD but a higher percentage of mild NAFLD obtained by Malik et al. 2007 (5). Hosseinpanath et al. (2007) found $82.9 \%$ had NAFLD, out of which $44.4 \%$ had mild, $43 \%$ had moderate and $12.6 \%$ had severe (6) which are comparable with the current study. On the other hand Cheah et al. (2013) found $44.2 \%$ of the respondents had NAFLD and among them half (52.9\%) had a moderate grade fatty liver in contrast with the current study (7).

There was no statistically significant $(p>0.05)$ difference with the grading of fatty liver with age. Although the mean age $(41.1 \pm 7.1)$ was higher in Grade 3 in this study (Table 2). The mean age of respondents with NAFLD in Cheah et al. (2013) study was 50 years, which is higher than the current study

(7). This may be explained by a bimodal age distribution of NAFLD by sex. In women, the prevalence of NAFLD peaks after age 50 years. Estrogens may be partially protective against steatosis (8). The higher mean age may be due to geographical variations, racial, ethnic differences, genetic causes, different lifestyle and increased life expectancy may have significant impacts to developed fatty liver (9).

In this study male to female ratio was almost 2:3 in the whole study subject (Table 3 ), which indicates that fatty liver is more common in female subject, which is similar with Chung et al. (2012) study, where they found $62.4 \%$ were female (10).

In the present study it was observed that the mean BMI was significantly $(\mathrm{p}<0.05)$ higher in grade $3(30.0 \pm 4.1)$ followed by grade $2(29.2 \pm 4.5)$ and grade $1(26.9 \pm 3.6)$ (table 4). The mean BMI of the whole study in Kirovski et al. (2010) found $26.4 \pm 5.0 \mathrm{~kg} / \mathrm{m}^{2}$ indicated a tendency to be slightly overweight as it is also observed in the general German population $(9,10)$. 
In the present study it was found that the mean FT3 and FT4 were significantly $(\mathrm{p}<0.05)$ reduced with increased grade of fatty liver and the mean TSH level was significantly $(\mathrm{p}<0.05)$ increased with increased grade of fatty liver (Table 5). In spearman's rank correlation test it was observed that no significant negative correlation $(r=-0.101$; $\mathrm{p}=0.175$ ) between FT3 and fatty liver of the study population (Figure 1), but a significant negative correlation $(\mathrm{r}=-0.277 ; \mathrm{p}=0.001)$ was observed between FT4 and fatty liver of the study population (Figure 2). On the other hand a significant positive correlation $\quad(\mathrm{r}=0.325 ; \mathrm{p}=0.001) \quad$ was observed between TSH and fatty liver of the study patients (Figure 3).

Pacifico et al. (2013) reported that elevated TSH concentrations were significantly associated with an increased prevalence of hepatic steatosis but no significant association in serum FT3 and FT4 levels. They also found a significant association between elevated TSH levels and metabolic variables (including hypertriglyceridemia and IR which are usually altered in fatty liver) (12). Carulli et al. (2013) also reported that serum TSH levels, in the NASH group were significantly $(p=0.017)$ greater compared to the steatosis group (13). This finding indicates that serum TSH concentration is increased with the severity of fatty liver disease.

Another population based study evaluated a relatively large numbers of healthy individuals in West Pomerania, a region of Northeast Germany showed that no consistent association of serum TSH and FT3 concentrations with hepatic steatosis. In contrast, serum FT4 concentrations were inversely associated with hepatic steatosis in men (odds ratio $=0.04)$ and women (odds ratio $=0.06)(14)$. Chung et al. (2012) also reported that FT4 were inversely associated with an increased prevalence of NAFLD after adjustment for risk factors. In this study it was observed that serum FT4 levels were significantly decreased with the grading of fatty liver (10).
A cross sectional study by Xu et al (2011) reported that NAFLD had higher serum TSH levels and lower FT4 levels, and the severity of NAFLD was negatively correlated with serum FT4 levels but positively correlated with TSH levels which is similar to this study. However FT3 was not statistically significant. A significant correlation between the serum TSH, FT4 level and metabolic syndrome parameters indirectly supported the relationship between thyroid function and NAFLD (15).

In this current study, out of 155 fatty liver subjects, 23 (14.8\%) were suffering from subclinical hypothyroidism, $4(2.6 \%)$ were suffering from hypothyroidism and rest were euthyroid (82.6\%). Among subclinical hypothyroid patients $6(7.3 \%)$ were in grade $1,13(26 \%)$ were in grade $2 \& 4(17.4 \%)$ were in grade 3 . All $4(17.4 \%)$ hypothyroid patients were in Grade 3 (17.4\%) (Table 6). In this study hypothyroidism was present only in grade 3 fatty liver which was the severe stage of NAFLD by USG. A cross sectional study by Pagadala et al (2012) reported that hypothyroidism was more frequent among patients with NAFLD ( $21 \%$ vs $9.5 \%$; p <0.01) compared to controls, and was higher in NASH patients than NAFLD patients without NASH ( $25 \%$ vs $12.8 \%$; $=0.03$ ) (16). Mazo et al. (2011) reported that prevalence of hypothyroidism was increased with the severity of fatty liver but not statistically significant (17).

Patients with hypothyroidism have abnormal lipid profiles mostly in the form of elevated serum levels of LDL (2). Elevated TSH level was significantly associated with hypertriglyceridemia (12) and hypertriglyceridemia increases the importation of triglyceride into liver and is therefore associated with development of fatty liver (9). Elevated serum makers of oxidative stress have been reported in patients with hypothyroidism and oxidative stress is one of the mechanisms involved in NAFLD (2). Hypothyroid patients have increased levels of leptin, a hormone which is increased in obesity and insulin resistance (18). All of these mechanisms which were important 
components of the metabolic syndrome favors association of hypothyroidism with NAFLD.

\section{CONCLUSION}

Fatty liver disease is one of the most common chronic liver disease. Prevalence and burden of this disease is increasing with time. On the other hand, incidence of thyroid disorders are also common. In this study it was found that FT4 was negatively and TSH was positively correlated with increasing grades of fatty liver and there is an increasing trend of hypothyroidism with the increasing grades of fatty liver. So a routine thyroid hormone test of all the newly diagnosed fatty liver patients may be helpful to evaluate the patients as well as planning for the management. On the other hand fatty liver may also be evaluated in all hypothyroid patients.

\section{REFERENCES}

1. Ramzan M, Ali I, Matin A. Sonographic Assessment of Hepatic Steatosis (Fatty Liver) in School Children of Dera Ismail Khan City (NWFP) Pakistan. Pakistan Journal of Nutrition 2009; 8(6):797-799.

2. Eshraghian A, Dabbaghmanesh $\mathrm{MH}$, Eshraghian H, Fattahi MR, Omrani GR. Nonalcoholic fatty liver disease in a cluster of Iranian population: Thyroid status and metabolic risk factors. Archives of Iranian Medicine 2013; 16(10):584-589.

3. LaBrecque DR, Abbas Z, Anania F, Ferenci P, Khan AG, Goh $\mathrm{K}$ et al. World Gastroenterology Organization Global Guidelines- Nonalcoholic Fatty Liver Disease and Nonalcoholic steatohepatitis. J Clin Gastroenterol 2014; 48(6):467-473.

4. Gardner CG, Richardson P, Wong C, Polavarapu N, Kemp G J, Cuthbertson D J. Hypothyroidism in a patient with nonalcoholic fatty liver disease. B.M.J 2011; 342: 168-170.

5. Malik A, Cheah PL, Hilmi IN, Chan SP, Goh KL. Nonalcoholic fatty liver disease in Malaysia: A demographic, anthropo $\neg$ metric, metabolic and histological study. Multiphase Pumping and Technologies Conference and Exhibition 2007; 8:58-64.

6. Hosseinpanah F, Rambod M, Sadeghi L. Predictors of NonAlcoholic Fatty Liver Disease in Type 2 Diabetes. Int J Endocrinol Metab 2007; 2:61-69.

7. Cheah WL, Lee PY, Chang CT, Mohamed HJ, Wong SL. Prevalence of ultrasound diagnosed non-alcoholic fatty liver disease among rural indigenous community of Sarawak and its association with biochemical and anthropometric measures. Southeast Asian J Trop Med Public Health 2013; 44( 2):309-317.

8. Fan JG, Saibara T, Chitturi S, Kim BI, Sung JJY, Chutaputti A. What are the risk factors and settings for non-alcoholic fatty liver dis $\neg$ ease in Asia-Pacific? J Gastroenterol Hepatol 2007; 22:794-800.

9. Kirovski G, Schacherer D, Wobser H, Huber H, Niessen C, Beer $\mathrm{C}$ et al. Prevalence of ultrasound-diagnosed nonalcoholic fatty liver disease in a hospital cohort and its association with anthropometric, biochemical and sonographic characteristics. Int J Clin Exp Med 2010; 3( 3): 202-210.

10. Chung GE, Kim D, Kim W, Yim JY, Park MJ, Kim YJ et al. Non-alcoholic fatty liver disease across the spectrum of hypothyroidism. J hepatol 2012; 57:150-156.

11. Hauner H, Bramlage P, Losch C, Steinhagen-Thiessen E, Schunkert H, Wasem $\mathrm{J}$ et al. Prevalence of obesity in primary care using different anthropometric measures-results of the German Metabolic and Cardiovascular Risk Project (GEMCAS). BMC Public Health 2008; 8: 282-290.

12. Pacifico L, Bonci E, Ferraro F, Andreoli G, Bascetta S, Chiesa C. Hepatic Steatosis and Thyroid Function Tests in Overweight and Obese Children. International Journal of Endocrinology 2013; 55:1-6.

13. Carulli L, Ballestri S, Lonardo A, Lami F, Violo E, Losi L et al. Is nonalcoholic steatohepatitis associated with a highthough-normal thyroid stimulation hormone level and lower cholesterol levels. Intern Emerg Med 2013; 8: 297-305.

14. Ittermann T, Haring R, Wallaschofski H, Baumeister SE, Nauck M, Dorr $M$ et al. Inverse association between serum free thyroxine levels and Hepatic steatosis: Results from the study of health in Pomerania. Thyroid foundation and dysfunction 2012; 22(6): 568-574.

15. Xu C, Xu L, Yu C, Miao M, Youming L. Association between thyroid function and nonalcoholic fatty liver disease in euthyroid elderly Chinese. Clinical Endocrinology 2011; 75: 240-246.

16. Pagadala MR, Zein CO, Dasarathy S, Yerian LM, Lopez R, McCullough AJ. Prevalence of Hypothyroidism in Nonalcoholic Fatty Liver Disease. Dig Dis Sci 2012; 57 : 528-534.

17. Mazo DF, Lima VM, Stefano JT, Rabelo F, Faintuch J, Oliveira, CP. Gluco-lipidic indices in treated hypothyroidism associated with nonalcoholic fatty liver disease. Arq Gastroenterol 2011; 48: 186 - 189.

18. Leonhardt U, Ritzel U, Schafer G, Becker W, Ramadori G. Serum leptin levels in hypo- and hyperthyroidism. J Endocrinol 1998; 157:75-79. 